\title{
A CHARACTERIZATION OF THE EQUICONTINUOUS STRUCTURE RELATION
}

\author{
BY \\ ROBERT ELLIS AND HARVEY KEYNES( $\left.{ }^{(}\right)$
}

\begin{abstract}
The main result in the paper is to show that in a large class of minimal transformation groups (including those with abelian phase groups, and point-distal transformation groups), the equicontinuous structure relation is precisely the regionally proximal relation. The techniques involved enable one to recover and extend the previously known characterizations. Several corollaries are indicated, among which the most important is a new criterion (which is easily applicable) for the existence of a nontrivial equicontinuous image of a given transformation group.
\end{abstract}

1. Introduction. A recurrent and interesting problem in topological dynamics is to determine the equicontinuous structure relation for an arbitrary minimal transformation group. In [4] and [11], it was shown that in the distal or point-distal case with a compact metric space, there is a nontrivial equicontinuous factor. In the case when the group is abelian, it was shown in [6] and independently in [8] that there are no equicontinuous factors precisely when the transformation group is weakly mixing. In this paper, we unite all the above results by showing that in a large class of minimal transformation groups (including all the above cases), the equicontinuous structure relation is precisely the regionally proximal relation. This result not only includes the above but provides either easily obtained extensions or alternate proofs of many results concerning this structure relation. For example, it follows in the metric case (and by an inverse limit argument to the quasi-separable case) that there is a nontrivial equicontinuous factor if some proximal cell is not dense.

The above characterization leads naturally to the study of the relationship between the two known characterizations of the equicontinuous structure relation found in [2] and [10]. Using the above characterization, one is able to derive a generalization of the characterization in [10] to the class of transformation groups considered in this paper. We note in passing that it is a relatively straightforward matter to show that $S=Q$ with the latter of these characterizations. Moreover, the discovery of both characterizations is not surprising, since in general there are relationships between them.

Received by the editors June 17, 1970 .

AMS 1969 subject classifications. Primary 5482, 5480.

Key words and phrases. Equicontinuous structure relation, minimal transformation groups, distal and point-distal transformation groups, abelian transformation groups, locally almost periodic transformation groups, distal extensions.

(1) Research of both authors supported by NSF Grant GP-8691.

Copyright (C) 1971, American Mathematical Society 
Finally, we consider an important type of extension of this result-namely, the "relativized" case (i.e., using relativized relations over a homomorphic image), and indicate some preliminary results. A future paper will be devoted to further results in this case.

The main tools of this paper will be the algebraic techniques developed in [2]. Thus, we will use freely throughout this paper the notation and results established in [2]. All transformation groups will be assumed minimal with a compact Hausdorff phase space and discrete group unless otherwise stated. Given $(X, T)$, recall that the proximal relation is $P=\bigcap\{\alpha T \mid \alpha$ index of $X\}$, the regionally proximal relation is $Q=\bigcap\{\operatorname{cls}(\alpha T) \mid \alpha$ index of $X\}$, and the equicontinuous structure relation $S$ is the smallest relation $R_{0}$ such that $\left(X \mid R_{0}, T\right)$ is equicontinuous. It is known [3] that $S$ is the smallest closed invariant equivalence relation containing $Q$; hence $S=Q$ is equivalent to asserting that $Q$ is an equivalence relation.

Finally, for completeness, we give a brief algebraic survey of the essential facts needed. Thus, $M$ will denote a fixed minimal right ideal in $\beta T$, the Stone-Čech compactification of the discrete group $T$. All elements $p, q, r, \ldots$ are in $M$, and $u$ will denote a fixed idempotent of $M$. Also, $(X, T)$ will denote a fixed minimal transformation group. Hence, $(X, T)$ has a representation as a $T$-subalgebra $\mathscr{A} \subset \mathfrak{A}(u)=\{f \in \mathscr{C}(\beta T) \mid f u=f\}$, i.e., $(|\mathscr{A}|, T) \simeq(X, T)$, and we fix one such representative. We consider the groups $G=M u$ and $A=\mathfrak{g}(\mathscr{A})=\{\alpha \in G \mid f \alpha=f(f \in \mathscr{A})\}$. With its $\tau=\tau(\mathscr{C})$ topology, $G$ is a compact $T_{1}$ group with unilaterially continuous multiplication and $A$ is a closed subgroup. We also will have occasion to use the $\tau(\mathscr{A})$-topology on $G$. Finally, we will consider the $T$-subalgebra

$$
\mathscr{K}=\{f \in \mathfrak{A}(u) \mid f t \in \mathfrak{A}(u)(t \in T)\} .
$$

Note that $f \in \mathscr{K}$ iff $f u t u=f u t(t \in T)$.

2. The characterization $S=Q$. The following lemma is direct and its proof will be omitted. Minimality is not necessary for its validity.

(2.1) LeMmA. If $x, y \in X$, then $(x, y) \in Q$ iff there exists $z \in X$ such that, given $U \in \mathscr{N}_{z}, V \in \mathscr{N}_{x}, W \in \mathscr{N}_{y}$, then $\{v t, w t\} \subset U$ for some $v \in V, w \in W, t \in T$.

Note that $\mathscr{N}_{\text {(.) }}$ will denote a neighborhood system.

Since $\mathscr{C}(M)=\mathfrak{A}(u), \mathscr{A}$ can be regarded as a subalgebra of $\mathscr{C}(M)$ and as such induces a topology $\mathscr{T}$ on $M$ which is compact but not necessarily Hausdorff. Remembering that $M$ is the universal minimal set for $T, \mathscr{T}$ may also be viewed as the topology on $M$ induced by the canonical restriction map $\pi:(M, T) \stackrel{\sim}{\rightarrow}$ $(|\mathscr{A}|, T)$. All topological notions such as closure, interior, and continuity will refer to $\mathscr{T}$ unless specified otherwise. Note that $((M, \mathscr{T}), T)$ again gives a minimal transformation group.

(2.2) Definition. Let $p, q \in M$. Then $(p, q) \in Q^{*}$ if, given any open set $R$ of $M$, $L \in \mathscr{N}_{p}, K \in \mathscr{N}_{q}$, then $\{l t, k t\} \subset R$ for some $l \in L, k \in K, t \in T$. 
The relation $Q^{*}$ corresponds to the regionally proximal relation of $((M, \mathscr{T}), T)$. We thus have

(2.3) Lemma. Let $p, q \in M$. Then $(p, q) \in Q^{*}$ iff $(p|\mathscr{A}, q| \mathscr{A}) \in Q=Q(|\mathscr{A}|)$.

Proof. Since $((M, \mathscr{T}), T)$ is minimal, we can restrict $R$ in $(2.2)$ to be an arbitrary neighborhood of a fixed point in $M$. The proof is completed by applying (2.1) and noting that $\pi$ is open.

(2.4) Corollary. $Q^{*}$ is a closed invariant symmetric subset of $M \times M$. Thus, if $(p, q) \in Q^{*}$, then $(p m, q m) \in Q^{*}(m \in M)$.

Let $U, V \subset T$. Then $[U, V]$ will denote the set $\{p \mid p \in M, U p \cap V \neq \varnothing\}$, and $h(U)$ the set $\{p \mid p \in M, U \in p\}$. Note here that we are using the ultra-filter interpretation of $\beta T$ [2, Chapter 8], whence $p q=\{A \mid A p \in q\}$ and $A p=\left\{t \mid A t^{-1} \in p\right\}$. Since $\mathscr{T}$-open neighborhoods of $p \in M$ have the form $\pi^{-1}(Z)$; it follows by [2, 11.13 and 11.14] that the set of all $h(U)$, where $h(U)$ is an $\mathscr{A}$-neighborhood of $p \in M$, forms a neighborhood base for $p$ in the $\mathscr{T}$-topology. We will frequently use these basic sets. Notice that here $h(U) \subset M$ always; this is a minor modification of the notation in [2].

Finally, let $p \in M$. Then there exists a unique idempotent $v$ of $M$ with $p v=p$. We let $\mathscr{M}_{p}$ be the collection of sets $[U, V]$, where $V \in v$, and $h(U)$ is a basic $\mathscr{T}$-neighborhood of $p$. Now $V v=V$ and $v=v e \in h(V)$ implies $e \in V v=V$. Thus $q=q e \in h(U)$ implies $q \in[U, V]$ and so $h(U) \subset[U, V]$. Hence, each $[U, V]$ is also a $\mathscr{T}$-neighborhood for $p$ in the case $V v=V$.

From this point, the procedure is as follows: We first show that under certain conditions on $\mathscr{A}$ (which are satisfied when $\mathscr{A} \subset \mathscr{K}$ ), $Q^{*}$ is an equivalence relation on $M$. From this, it easily follows that $Q$ is an equivalence relation and hence $S=Q$. A key set will be $\bigcap\left\{\right.$ cls $\left.N \mid N \in \mathscr{M}_{q}\right\}$, for $q \in M$. Since it occurs so frequently, we denote it by $[q]$. Now to show that $Q^{*}$ is an equivalence relation, it will suffice to show that $p Q^{*}=[p u](p \in M)$. As a by-product of this fact, we will later show that $p Q^{*}=[p](p \in M)$ and then derive a generalization of [10].

To pinpoint what is involved in the proof (and thus to indicate where generalizations are necessary to prove the result in arbitrary transformation groups), we consider the following two statements:

I. $[p] \subset(p \omega) Q^{*}(p \in M, \omega \in J$, the idempotents in $M)$.

II. $\alpha Q^{*} \subset[\alpha](\alpha \in G)$.

Then I is always true and with conditions on $\mathscr{A}$, II holds and $Q^{*}$ is an equivalence relation.

(2.5) Lemma. Let $U, V \subset T$ and $t \in T$. Then $[U, V]=[U t, V t]$.

Proof. Let $p \in M$. Then $p \in[U, V]$ iff $U p \cap V \neq \varnothing$ iff $U p t \cap V t \neq \varnothing$. Since $U p t=U t p[2,3$ of 8.10], $p \in[U, V]$ iff $U t p \cap V t \neq \varnothing$ iff $p \in[U t, V t]$. 
(2.6) Lemma. Let $v \in J$ and $U \in v$. Then there exists $V \in v$ with $V v=V$ and $h(V) \subset h(U)$.

Proof. Set $V=U v$. Then $U \in v=v^{2}$ implies that $V=U v \in v$. Moreover, $p \in h(U v)$ iff $U v \in p$ iff $U \in v p=p$ iff $p \in h(U)$. Thus, $h(V)=h(U)$.

(2.7) Lemma. Let $U \subset T$ and $p \in M$ with $U p \neq \varnothing$. Then $U q \neq \varnothing(q \in M)$.

Proof. Let $q \in M$. Then there exists $r \in M$ with $q r=p$. Since $U q=\varnothing$ implies $U p=(U q) r=\varnothing$, the result follows.

(2.8) Lemma. Let $p \in M$. Then $[p]=[p w](w \in J)$.

Proof. Let $x \in[p], h(W)$ a neighborhood of $x, h(U)$ a neighborhood of $p w$ and $V \in w$. Also, let $v t_{n} \rightarrow w$ in the usual topology of $M$, where $p v=p$.

Now $p t_{n}=p v t_{n} \rightarrow p w$ in the usual topology and hence the $\mathscr{T}$-topology on $M$. Hence $v t \in h(V)$ and $p t \in$ int $h(U)$ for some $t$. Then $h\left(U t^{-1}\right)=h(U) t^{-1}$ is a neighborhood of $p$ and $V t^{-1} \in v$. Thus $x \in$ cls $\left[U t^{-1}, V t^{-1}\right]$ and consequently $\varnothing \neq h(W)$ $\cap\left[U t^{-1}, V t^{-1}\right]=h(W) \cap[U, V]$ by (2.5). Thus $x \in[p w]$, and so $[p] \subset[p w]$.

Now replace $p$ by $p w$ and $w$ by $v$ in the above argument. This yields $[p w] \subset[p w v]$ $=[p v]=[p]$. The result follows.

The next result is the proof of $I$.

(2.9) Proposition. Let $p \in M$. Then $[p] \subset(p w) Q^{*}(w \in J)$.

Proof. We first show that for any $r \in M,[r] \subset r Q^{*}$. Let $v \in J$ with $r v=r, q \in[r]$, and $U, W \subset T$ such that $h(U) \in \mathscr{N}_{q}$ and $h(W) \in \mathscr{N}_{r}$. Since $h(W)$ is also a neighborhood of $r$ in the ordinary topology, there exists $V \in v$ with $r h(V) \subset h(W)$. By (2.6), we can assume $V v=V$. Then $[W, V] \in \mathscr{M}_{r}$, and hence $h(U) \cap[W, V] \neq \varnothing$. If $m \in h(U) \cap[W, V]$, then $m t \in h(W)$ for some $t \in V$. Since $v t \in h(V)$, then $r t \in h(W)$. It then follows by the comment in $(2.3)$ that $(r, q) \in Q^{*}$.

Finally, if $w \in J$, then $[p]=[p w] \subset(p w) Q^{*}$, using (2.8).

We now put a condition on $\mathscr{A}$ that together with II will show that $Q^{*}$. is an equivalence relation. Recall that $\mathscr{E} \subset \mathfrak{A}(u)$ is the subalgebra of almost periodic functions and $E$ its group. Now consider the $\tau(\mathscr{A})$-topology on $G$ and set $H(G, \mathscr{A})$ $=\bigcap\{$ cls $N \mid N$ a $\tau(\mathscr{A})$-neighborhood of $u\}$ (see [2, 11.9-11.11] for the definition of the $\tau(\mathscr{A})$-topology). Note the resemblance of $H(G, \mathscr{A})$ to $[u]$. If $E \subset H(G, \mathscr{A})$, then it follows that given $\gamma, \delta \in G$, then $(\gamma, \delta) \in Q^{*}$ iff $\gamma^{-1} \in A E[2,14.17$ and 14.18].

(2.10) TheOREM. Suppose that $E \subset H(G, \mathscr{A})$ and $\alpha Q^{*} \subset[\alpha](\alpha \in G)$. Then

1. $\alpha Q^{*}=[\alpha](\alpha \in G)$.

2. $(\alpha, \beta) \in Q^{*}$ implies $\alpha Q^{*}=\beta Q^{*}(\alpha, \beta \in G)$.

3. $p Q^{*}=(p u) Q^{*}(p \in M)$.

4. $Q^{*}$ is an equivalence relation on $M$. 
Proof. 1. This follows from the hypotheses and (2.9).

2. Let $x \in \alpha Q^{*}$. Then $(x u, \alpha) \in Q^{*}$ by (2.4). Thus $(x u) \alpha^{-1}, \alpha \beta^{-1} \in A E$ and, since $A E$ is a group, $(x u) \beta^{-1} \in A E$. This means that $(x u, \beta) \in Q^{*}$ whence $\beta \in(x u) Q^{*}$ $=[x u]$ by 1 . If $v \in J$ with $x v=x$, then $[x u] \subset(x u v) Q^{*}=x Q^{*}$ by (2.9). Hence $\beta \in x Q^{*}$ and thus $x \in \beta Q^{*}$ by (2.4). We then have $\alpha Q^{*} \subset \beta Q^{*}$; the reverse inclusion follows similarly.

3. First $(p u) Q^{*}=[p u] \subset p Q^{*}$ by (2.9). On the other hand, let $x \in p Q^{*}$. Then $(x u, p u) \in Q^{*}$ whence $p u \in x u Q^{*}=[x u] \subset x Q^{*}$. Thus $x \in(p u) Q^{*}$.

4. It suffices to show that if $x \in y Q^{*}$ then $x Q^{*}=y Q^{*}$. Let $x \in y Q^{*}$. Then $(x u, y u) \in Q^{*}$, and so $x Q^{*}=(x u) Q^{*}=(y u) Q^{*}=y Q^{*}$ using 2 and 3 . The proof is completed.

The next series of results show that when $\mathscr{A} \subset \mathscr{K}$ then $\alpha Q^{*} \subset[\alpha](\alpha \in G)$. Since by [2, Proposition 15.13], $E=H(G, \mathscr{K})$, it follows that $\mathscr{A} \subset \mathscr{K}$ implies $E=H(G, \mathscr{K})$ $\subset H(G, \mathscr{A})$. Thus, the assumption $\mathscr{A} \subset \mathscr{K}$ is sufficient to guarantee that (2.10) holds. Note also that, since $[u] \cap G \subset u Q^{*} \cap G=A E \subset A H(G, \mathscr{A})=H(G, \mathscr{A}),[U, V] \in M_{u}$ implies $[U, V] \cap G=(U, V)$ is a $\tau(\mathscr{A})$-neighborhood of $u[2,11.14 .1]$, and the $\mathscr{T}$-topology on $G$ contains the $\tau(\mathscr{A})$-topology by $[2,11.15]$, then $[u] \cap G=H(G, \mathscr{A})$ under this assumption.

In the following, a subscript of $\mathscr{A}$ will mean the operation with respect to the $\tau(\mathscr{A})$-topology on $G$.

(2.11) Lemma. Let $\mathscr{A} \subset \mathscr{K}, p \in M, R$ a neighborhood of $p$ and

$$
L=\operatorname{int}_{\mathscr{A}} \operatorname{cls}_{\mathscr{A}}(R \cap G) .
$$

Then $p u \in \operatorname{cls}_{\mathscr{A}} L$.

Proof. Since $\mathscr{A} \subset \mathscr{K}, u t u|\mathscr{A}=u t| \mathscr{A}(t \in T)$, whence $\{\alpha|\mathscr{A}| \alpha \in G\}$ is dense in $|\mathscr{A}|$. Hence $G$ is $\mathscr{T}$-dense in $M$.

Let $N$ be an open subset of $M$. Then there exists a finite subset $F$ of $T$ such that $N F=M$.

Since cls $G=M, N$ open implies $N \subset \operatorname{cls}(N \cap G)$ whence $G \subset M \subset(\operatorname{cls}(N \cap G)) F$. Let $\alpha \in G$. Then $\alpha u=\alpha=g t$ with $g \in \operatorname{cls}(N \cap G)$ and $t \in F$. Hence $\alpha u t^{-1}=g$ and so $\alpha u t^{-1} u=g u$. Now $g \in \operatorname{cls}(N \cap G)$ implies that $g u \in \operatorname{cls}_{\mathscr{A}}(N \cap G)$ [2, 11.15]. Consequently $G \subset\left(\operatorname{cls}_{\mathscr{A}}(N \cap G)\right) H$ where $H=\left\{\left(u t^{-1} u\right)^{-1} \mid t \in F\right\}$. Since $H$ is finite and each set is closed (right multiplication is a $\tau(\mathscr{A})$-homeomorphism $[2,11.17]$ ), this implies that int $\mathscr{A} \operatorname{cls}_{\mathscr{A}}(N \cap G) \neq \varnothing$.

Now let $K$ be a neighborhood of $p$ and set $N=\operatorname{int}_{\mathscr{A}}(R \cap K)$. Then

$$
\varnothing \neq \operatorname{int}_{\mathscr{A}} \operatorname{cls}_{\mathscr{A}}(N \cap G) \subset L .
$$

Hence $\operatorname{cls}_{\mathscr{A}}(N \cap G) \cap L \neq \varnothing$ and so $N \cap G \cap L \neq \varnothing$ since $L$ is $\tau(\mathscr{A})$-open. Then $N \subset K$ implies $K \cap L \neq \varnothing$. This implies that $p \in \operatorname{cls} L$, whence $p u \in \operatorname{cls}_{\mathscr{A}} L$ as before. The result follows. 
(2.12) Lemma. Let $\mathscr{A} \subset \mathscr{K}, N$ a $\tau(\mathscr{A})$-open subset of $G$, and $L=\operatorname{cls}_{\mathscr{A}} N$. Then $A E L=L$.

Proof. Since $E \subset H(G ; \mathscr{A})$, then $E \subset \operatorname{cls}_{\mathscr{A}} R$ for every $\tau(\mathscr{A})$-neighborhood $R$ of $u$. Since right multiplication is a $\tau(\mathscr{A})$-homeomorphism, then $E_{\alpha} \subset L(\alpha \in N)$. Thus $E N \subset L$. Since $E$ is a normal subgroup of $G, E N=N E$. Thus if $\beta \in E, N \beta \subset L$ implies $L \beta \subset L$, whence $L E=E L \subset L$. Moreover, it follows immediately from the definitions involved that $A L=L$. Hence $A E L=E A L=E L \subset L$, and the proof is completed.

(2.13) THEOREM. Let $\mathscr{A} \subset \mathscr{K}$. Then

1. $Q^{*}$ is an equivalence relation on $M$.

2. $Q$ is an equivalence relation on $|\mathscr{A}|=X$.

3. $Q=S$.

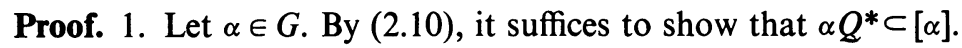

Let $p \in \alpha Q^{*}, R$ a neighborhood of $p, h(U)$ a neighborhood of $\alpha$ and $V \in u$.

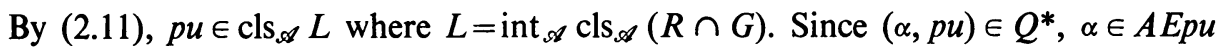
$\subset A E \operatorname{cls}_{\mathscr{A}} L=\operatorname{cls}_{\mathscr{A}} L$ by (2.12).

Now $(U, V)=[U, V] \cap G$ is a $\tau(\mathscr{A})$-neighborhood of $\alpha[2,11.14]$. Let $N$ be a $\tau(\mathscr{A})$-open set with $\alpha \in N \subset(U, V)$. Then $N \cap L \neq \varnothing$. This implies that $[U, V] \cap R$ $\neq \varnothing$. Hence $p \in \operatorname{cls}[U, V]$, from which it follows that $p \in[\alpha]$.

2. Let $(x, y),(y, z) \in Q$. Choose $p, q, r \in M$ such that $p|\mathscr{A}=x, q| \mathscr{A}=y, r \mid \mathscr{A}=z$. Then $(p|\mathscr{A}, q| \mathscr{A}),(q|\mathscr{A}, r| \mathscr{A}) \in Q$ and $(p, q),(q, r) \in Q^{*}$ by (2.3). Then $(p, r) \in Q^{*}$ by 1 and $(p|\mathscr{A}, r| \mathscr{A})=(x, z) \in Q$ by (2.3) again. The result follows.

We now note that, when $T$ is abelian, $u t u=u u t=u t(t \in T)$, whence $\mathscr{K}=\mathfrak{A}(u)$, and thus (2.13) is always applicable. For the nonabelian case, we can apply (2.13) when $\mathscr{A}$ is point-distal [11, p. 481] (for an equivalent algebraic definition, cf. [2, Notes to Chapter 15, 3.3]). To see this, consider $\mathscr{L}=\{f \in \mathfrak{A}(u) \mid f v=f(v$ a minimal idempotent in $\beta T)\}$ and note that $\mathscr{A} \subset \mathscr{L}$; in fact, $\mathscr{L}$ is the algebra corresponding to the universal minimal point-distal flow ([7, p. 301], cf. [2, 2 of 15.14]). Now we claim $\mathscr{L} \subset \mathscr{K}$. For let $t \in T$. Then $\left(t u t^{-1}\right)^{2}=t u t^{-1} t u t^{-1}=t u^{2} t^{-1}=t u t^{-1}$, whence tut ${ }^{-1}$ is a minimal idempotent. Thus if $f \in \mathscr{L}$, then $f u t u t^{-1}=f t u t^{-1}=f=f u$ and futu $=$ fut. This means that $f \in \mathscr{K}$.

Finally, we discuss the two aforementioned characterizations. In [10, Theorem 1.1], the following was proved: Let $T$ be abelian and for $x \in X, U \in \mathcal{N}_{x}$, set

$$
N(x, U)=\{t \mid x t \in U\}
$$

Then

(A) $x S=\bigcap\left\{\right.$ cls $\left.x N(x, U) N(x, U)^{-1} \mid U \in \mathscr{N}_{x}\right\}$ where $x S=\{y \mid(x, y) \in S\}$.

One should note that when (A) holds, a short proof that $S=Q$ is obtained.

(2.14) Proposition. If (A) holds, then $S=Q$. 
Proof. Let $y \in x S=\bigcap\left\{\right.$ cls $\left.x N(x, U) N(x, U)^{-1} \mid U \in \mathscr{N}_{x}\right\}$. Then given $W \in \mathscr{N}_{y}$, $U \in \mathscr{N}_{x}$, there exists $w \in W, t, s \in T$ for which $w t=x s$ and $\{x t, x s\}=\{x t, w t\} \subset U$. Letting $z=x$ and setting $v=x$ in $(2.1)$, we have $(x, y) \in Q$. Since the other inclusion is obvious, the result follows.

The natural question occurs as to whether (A) is extendable to the case $\mathscr{A} \subset \mathscr{K}$. The content of the next few results is to provide this generalization of (A) to a slightly smaller algebra.

(2.15) Lemma. Let $\mathscr{A} \subset \mathscr{K}$. Then $[p]=p Q^{*}(p \in M)$.

Proof. Let $p \in M$. Then $[p]=[p u]=(p u) Q^{*}=p Q^{*}$ by (2.8), 1 of (2.13), and 3 of (2.10).

We now let $\mathscr{K}_{1}=\{f \in \mathfrak{A}(u) \mid f v t v=f v t$ ( $v$ minimal idempotent in $\left.M)\right\}$. Note that if $f \in \mathfrak{A}(u)$, then $f \in \mathscr{K}$ iff $f v t \in \mathfrak{A}(v)\left(v^{2}=v \in M, t \in T\right)$.

(2.16) TheOREM. Let $\mathscr{A} \subset \mathscr{K}_{1}$ and $x \in X=|\mathscr{A}|$. Then

$$
x S=\bigcap\left\{\operatorname{cls} x N(x, W) N(x, W)^{-1} \mid W \in \mathscr{N}_{x}\right\} .
$$

Proof. Let $p \in M$ with $p v=p, v^{2}=v \in M$, and $p \mid \mathscr{A}=x$. Then $x S=x Q=\pi\left(p Q^{*}\right)$ $=\pi([p])=\pi([p v]) \subset \bigcap$ cls $\pi[U, V]$ by $(2.10),(2.3),(2.15)$ and (2.8). Since always $\bigcap\left\{\right.$ cls $\left.x N(x, W) N(x, W)^{-1} \mid W \in \mathscr{N}_{x}\right\} \subset x S$, the assertion will follow if we show $\bigcap$ cls $\pi[U, V] \subset \bigcap$ cls $x N(x, W) N(x, W)^{-1}$.

Let $W \in \mathscr{N}_{x}$. Then $\pi h(U) \subset W$, where $h(U)$ is a $\mathscr{T}$-neighborhood of $p v$. Hence $p v \in \operatorname{int} h(U)$ and the fact that the set of $h(W) \in \mathscr{N}_{p v}$ is basic implies that $h(L) \subset \operatorname{int} h(U)$ with $h(L) \in \mathscr{N}_{p v}$. By (2.6), there exists $V \in v$ with $V v=V$ and $p h(V) \subset h(U)$. Let $q \in[L, V]$. Then there exists $t \in V$ with $t \in h(L) \subset$ int $h(U)$. Then, $K t \subset$ int $h(U), K$ a neighborhood of $q$. Thus $K \subset[U, V]$ and $q \in$ int $[U, V]$. So $\pi[L, V] \subset$ $\pi$ int $[U, V] \subset$ int $\pi[U, V]$, and

$$
\text { cls } \pi[L, V] \subset \text { cls int } \pi[U, V] \text {. }
$$

We will complete the proof by showing cls int $\pi[U, V] \subset \operatorname{cls} x N(x, W) N(x, W)^{-1}$.

Let $\pi(p v s v) \in$ int $\pi[U, V]$ for some $s \in T$. Then $r \in[U, V]$ and $\pi(r)=\pi(p v s v)$ for some $r \in M$. Thus, $r t \in h(U)$ for some $t \in V$. Now $t \in V=V v$ implies $v t \in h(V)$ and thus $p v t \in h(U)$. Then $\pi(r t)=\pi(r) t=\pi(p v s v) t=\pi(p v s) t=\pi(p v) s t \in \pi(h(U))$ and $s t \in N(x, \pi h(U))$. Moreover, $t \in N(x, \pi h(U))$. Thus,

$$
\begin{aligned}
\pi(p v s v) & =\pi(p v s v t) t^{-1} \\
& =\pi(p v)(s t) t^{-1} \in x N(x, \pi h(U)) N(x, \pi h(U))^{-1} \subset x N(x, W) N(x, W)^{-1} .
\end{aligned}
$$

Since $\{\pi(p v s v) \mid s \in T\}$ is dense in $X$, cls int $\pi[U, V] \subset \operatorname{cls} x N(x, W) N(x, W)^{-1}$, and the desired assertion follows.

If one realizes that when $T$ is abelian $(U, V)=[U, V] \cap G=h(U) h(V)^{-1} \cap G$, the conclusion of (2.16) is not unexpected. Indeed, the assumption $\mathscr{A} \subset \mathscr{K}_{1}$ "abelianizes" $T$ in the $\mathscr{T}$-topology. Also, the proof given after (2.13) applied to any minimal idempotent $v \in M$ shows that $\mathscr{L} \subset \mathscr{K}_{1}$. Moreover, $T$ abelian implies $\mathscr{K}_{1}=\mathfrak{A}(u)$. 
So (2.16) holds in both the abelian and point-distal case.

We finally recall the characterization of $S$ due to Ellis [2, 14.23]: If $\mathscr{B} \subset \mathscr{A} \subset \mathfrak{A}(u)$, then $\mathscr{R}(\mathscr{A}: \mathscr{B})=\{(p|\mathscr{A}, q| \mathscr{A}) \mid p, q \in M$ and $p|\mathscr{B}=q| \mathscr{B}\}$. We frequently write $x, y, \ldots$, for $p|\mathscr{A}, q| \mathscr{A}, \ldots$ Then if $E \subset H(G, \mathscr{A})$, we have that

$$
\begin{aligned}
S & =\mathscr{R}(\mathscr{A}: \mathscr{A} \cap \mathscr{E}) \\
& =\left\{(x, y) \mid(x w, y w) \in Q\left(w^{2}=w \in K, K \text { minimal ideal in } \beta T\right)\right\} .
\end{aligned}
$$

With regard to the various implications without restrictions on $\mathscr{A}$, we have already noted that (A) implies $Q=S(2.14)$. We also have

(2.17) LEMMA. If $S=Q$, then (B) holds.

Proof. Denote $\{(x, y) \mid(x w, y w) \in Q$ (w minimal idempotent in $\beta T)\}$ by $R$. Then $Q$ closed invariant implies $Q \subset R$ whence $S \subset R$. Now let $(x, y) \in R$. Then $y w=y$ for some $w^{2}=w \in M$. Now $(x, x w) \in P \subset S$, whence $(x, y)=(x, x w)(x w, y w) \in S^{2}=S$. Thus, $R \subset S$.

It is not known if the converse holds. However, a closely related result is true.

(2.18) LEMMA. (B) holds iff $S=P Q$ (the product of relations).

Proof. Suppose (B) holds. We need only show $S \subset P Q$. Let $(x, y) \in S=R$, using notation of (2.17). Choose $v^{2}=v \in M$ such that $y v=y$. Then

$$
(x v, y v)=(x v, y) \in Q
$$

by assumption. Moreover, $(x, x v) \in P$. Thus $(x, y)=(x, x v)(x v, y) \in P Q$.

Now suppose $S=P Q$ and $(x, y) \in S$. Then $(x, z) \in P,(z, y) \in Q$ for some $z \in|\mathscr{A}|$. Since for some minimal ideal $K, z=x w$ with $w^{2}=w \in K$, then $(x w, y) \in Q$ and $\left(x w_{1}, y w_{1}\right)=(x w, y) w_{1} \in Q$ for all $w_{1}^{2}=w \in K$. Now for any minimal ideal $N$ and $w^{2}=w \in N, S$ closed invariant implies $(x w, y w) \in S$. Applying the above to $(x w, y w)$, then $\left(x w w_{1}, y w w_{1}\right) \in Q$ for all $w_{1}^{2}=w_{1}$ in some minimal ideal $K$. Since $w w_{1}=w$ for some $w_{1}^{2}=w_{1} \in K$, then $(x w, y w) \in Q$ and hence $(x, y) \in R$. The other inclusion follows as in (2.17), and the proof is completed.

The primary tool used to show that (B) holds is the assumption that $E \subset H(G, \mathscr{A})$. The authors conjecture that if $E \subset H(G, \mathscr{A})$, then $S=Q$ and all assertions are equivalent. So if one looks to generalize these assertions by proving $E \subset H(G, \mathscr{A})$ the most natural path would be to use (2.10). However, if (B) could be shown to hold, then one would have a weaker but still useful characterization of $S$. To see one case when (B) does hold, recall that if $\mathscr{B} \subset \mathscr{A}$, then $\mathscr{A}$ is a proximal extension of $\mathscr{B}$ if $\mathscr{A} \subset \mathfrak{A}$ (B) or, equivalently, $A=B$, where $A$ and $B$ are the groups of $\mathscr{A}$ and $\mathscr{B}$ respectively. It follows from $[2,13.16]$ that another characterization is

$$
R(\mathscr{A}: \mathscr{B}) \subset P(\mathscr{A}) \text {. }
$$

(2.19) LEMMA. If $\mathscr{A}$ is a proximal extension of $\mathscr{B}$, and $Q(\mathscr{B})=S(\mathscr{B})$, then $S(\mathscr{A})$ $=P^{2}(\mathscr{A}) Q(\mathscr{A})$. 
Proof. Let $(x, y) \in S(\mathscr{A})$. Then $(x|\mathscr{B}, y| \mathscr{B}) \in S(\mathscr{B})=Q(\mathscr{B})$. By [2, 14.2], there exists $(w, z) \in Q(\mathscr{A})$ with $w|\mathscr{B}=x| \mathscr{B}, z|\mathscr{B}=y| \mathscr{B}$. Then $(x, w),(z, y) \in P(\mathscr{A})$ by assumption, whence $(x, y)=(x, w)(w, z)(z, y) \in P(\mathscr{A}) Q(\mathscr{A}) P(\mathscr{A})$. Since it is direct to verify that $Q(\mathscr{A}) P(\mathscr{A})=P(\mathscr{A}) Q(\mathscr{A})$, the desired result obtains.

One consequence of (2.19) is that if $\mathscr{A}$ is a proximal extension of $\mathscr{B} \subset \mathscr{K}$ (whence $\mathscr{A} \subset \mathfrak{A}(K), K$ the group of $\mathscr{K})$, then $S(\mathscr{A})=P^{2}(\mathscr{A}) Q(\mathscr{A})$.

(2.20) Corollary. Suppose that $P(\mathscr{A})$ is closed. Then $S(\mathscr{A})=P(\mathscr{A}) Q(\mathscr{A})$.

Proof. If $\mathscr{A} \cap \mathscr{D}=\mathscr{B}$, then $P(\mathscr{A})$ closed means that $\mathscr{A}$ is a proximal extension of $\mathscr{B}$. Since $Q(\mathscr{B})=S(\mathscr{B})$ by $(2.13)$, then $S(\mathscr{A})=P^{2}(\mathscr{A}) Q(\mathscr{A})=P(\mathscr{A}) Q(\mathscr{A})$, noting that $P(\mathscr{A})$ is an equivalence relation.

3. Consequences of $S=Q$. In $\S 3$, we assume that $\mathscr{A} \subset \mathscr{K}$. When $X$ is metric, the characterization that $S=X \times X$ iff $x P=\{y \mid(x, y) \in P\}$ is dense for some $x \in X$ (or, equivalently, for a comeager set of $x \in X$ ) follows easily from [6, Remark 3.2]. For if $x P$ is dense for some $x \in X$, then $x S=X$ and $S=X \times X$; the other way comes from the above reference. We improve this result considerably by showing

(3.1) Proposition. Let $X$ be metric. Then $S=X \times X$ iff $x P$ is dense for every $x \in X$.

Proof. As noted above, $x P$ dense $(x \in X)$ implies $S=X \times X$; this holds independently of the metric assumption. Conversely, if $S=X \times X$, then $X=x S=x Q=$ $\bigcap\{x($ cls $(\alpha T)) \mid \alpha$ open index of $X\}=\bigcap\{$ cls $(x(\alpha T)) \mid \alpha$ open index of $X\}$ (since $p Q^{*}=[p]$ by (2.8) and (2.10)). Choosing a countable uniformity base $\left\{\alpha_{i} \mid i \geqq 1\right\}$ and noting that $x\left(\alpha_{i} T\right)$ is dense and open $(i \geqq 1)$, then $x P=\bigcap x\left(\alpha_{i} T\right)$ is dense.

Recall [5] that $\mathscr{A}$ is quasi-separable if $H=\{f \mid f \in \mathscr{A}$ and $|[f]|$ is metric $\}$ is dense in $\mathscr{A}$ or, equivalently, $X=|\mathscr{A}|=$ inv $\lim _{\alpha}\left|\mathscr{A}_{\alpha}\right|,\left|\mathscr{A}_{\alpha}\right|$ compact metric.

(3.2) Theorem. If $\mathscr{A}$ is quasi-separable, $S=X \times X$ iff $x P$ is dense $(x \in X)$.

Proof. We need only show $S=X \times X$ implies $x P(\mathscr{A})$ is dense $(x \in X)$. Suppose there exists $p \in M$ for which $p \mid \mathscr{A}=x$ and cls $(x P(\mathscr{A})) \neq|\mathscr{A}|$. Then $U=|\mathscr{A}|-$ cls $(x P(\mathscr{A}))$ is a nonempty open subset of $|\mathscr{A}|$. Choose a nonempty open subset $V$ of $|\mathscr{A}|$ such that $\bar{V} \subset U$, and $f \in \mathscr{A}$ for which $f|\bar{V} \equiv 0, f|$ cls $(x P) \equiv 1$. There exists $g \in H$ such that $g|\bar{V}<1 / 4, g| \operatorname{cls}(x P(\mathscr{A}))>3 / 4$. Let $\mathscr{B}=[g]$, and $Y=|\mathscr{B}|$. Then $Y$ is metrizable and we have $\phi:(|\mathscr{A}|, T) \stackrel{\sim}{\rightarrow}(|\mathscr{B}|, T)$ induced by $\mathscr{B} \subset \mathscr{A}$. We now claim that cls $(y P(\mathscr{B})) \neq|\mathscr{B}|$, if $y=p \mid \mathscr{B}$. For by minimality, int $\phi(V)=(V \mid \mathscr{B})^{0} \neq \varnothing$. Moreover, $g \in \mathscr{C}(Y)$ with $g|\phi(V)<1 / 4, g| y P(\mathscr{B})>3 / 4$, since $x P(\mathscr{A}) \mid \mathscr{B}=y P(\mathscr{B})$. Then $\phi(V)^{0} \cap y P(\mathscr{B})=\varnothing$ and cls $(y P(\mathscr{B})) \neq Y$. But $S(X)=X \times X$ implies $S(Y)=$ $Y \times Y$, as $\phi \times \phi(Q(X)) \subset Q(Y)$. This contradicts (3.1), and the result follows.

It follows by the remarks after (2.12) and (3.2) that when $(X, T)$ is point-distal (or distal), there is a nontrivial equicontinuous factor. A slight generalization is obtained with a local notion of distality, namely, by assuming that some point 
$x \in X$ is isolated in $x P$. One special case, of course, is when $x P$ is finite for some $x \in X$. Thus, if $(|\mathscr{B}|, T)$ is point-locally-distal with $\mathscr{B} \subset \mathscr{K}, \mathscr{B}$ quasi-separable, then $(|\mathscr{B}|, T)$ has a nontrivial equicontinuous factor. If $|\mathscr{B}|$ is finite, the assertion is obvious; if $|\mathscr{B}|$ is infinite, then minimality of $|\mathscr{B}|$ precludes a locally-distal point $x$ being isolated in $|\mathscr{B}|$. Hence, if $U \in \mathscr{N}_{x}$ with $x P \cap U=\{x\}$, then $V=U-\{x\}$ is a nonempty open set with $V \cap x P=\varnothing$. The assertion follows by (3.2). Finally, if $|\mathscr{B}|$ is metric and $x P$ is meager, then $x P$ is not dense (otherwise comeagerness would follow), and (3.1) is applicable.

It is known that when $T$ is abelian, $(X, T)$ is weakly-mixing iff $(X, T)$ has no nontrivial equicontinuous factor [6, Corollary 2.11] ([8, Proposition 1.11] for nonmetric case). This result also follows immediately from (2.12). For it is a relatively straightforward matter to show that $(X, T)$ is weakly mixing iff $Q=X \times X$ $[2,15.15]$; the other proofs revolved about showing $S=X \times X$ implies $Q=X \times X$. But this is now immediate. Extensions to arbitrary $\mathscr{A} \subset \mathscr{K}$ would follow if one could prove that $Q=X \times X$ implies that $(X, T)$ is weakly mixing in this case.

The next result is a collection of observations about $X$ which follow easily from (2.12).

(3.3) REMARK. Let $(Y, T)$ be minimal with $|\mathscr{B}|=Y, \mathscr{B} \subset \mathscr{K}$. Then

1. $S(X \times Y) \simeq S(X) \times S(Y)$ and $X \times Y \mid S(X \times Y) \simeq(X / S(X)) \times(Y \mid S(Y))$.

2. If $\phi:(X, T) \stackrel{\sim}{\rightarrow}(Y, T)$, then $\phi S(X)=S(Y)$, with obvious notation.

3. If $(X, T)=\operatorname{inv} \lim \left(X_{\alpha} T\right)$, then $S(X)=\bigcap \pi_{\alpha}^{-1} S\left(X_{\alpha}\right)$, where $\pi_{\alpha}:(X, T) \stackrel{\sim}{\rightarrow}\left(X_{\alpha} T\right)$ is canonical.

Proof. 1. We again have in both cases that the almost periodic points are dense in the product. With this assumption, it follows that if $M(X)$ is the regionally syndetically proximal relation [1, Definition 1], then $Q(X)=M(X)$. For choose $(w, z) \in Q(X), \alpha$ an open index and $U \in \mathscr{N}_{(w, z)}$. Then $U t \cap \alpha=V \neq \varnothing$ for some $t$. Choose an almost periodic point $(w, z) \in U$ with $\left(w_{1}, z_{1}\right) t \in V$. Then $\left(w_{1}, z_{1}\right) t A$ $\subset V \subset \alpha$ for some syndetic set $A$, and $(w, z) \in M(X)$. The other inclusion always holds.

Now $Q(X) \times Q(Y)=M(X) \times M(Y)=M(X \times Y) \subset Q(X \times Y)$ by [1, Theorem 4]. Since $Q(X \times Y) \subset Q(X) \times Q(Y)$ always holds, we have $Q(X \times Y)=Q(X) \times Q(Y)$ $=S(X) \times S(Y)$ by (2.12). Hence, $Q(X \times Y)$ is an equivalence relation and $Q(X \times Y)$ $=S(X \times Y)$, from which the result follows.

2. It is shown in $[2,14.2]$ that $\phi \times \phi Q(X)=Q(Y)$.

3. By 2 , we have that $S(X) \subset \bigcap \pi_{\alpha}^{-1}\left(S\left(X_{\alpha}\right)\right)$. Now it is a straightforward verification that $\bigcap \pi_{\alpha}^{-1}\left(Q\left(X_{\alpha}\right)\right) \subset Q(X)$. The result follows by applying (2.12).

An immediate consequence of 3 of (3.3) is that an inverse limit of weakly mixing minimal transformation groups with abelian $T$ is weakly mixing.

Recall $[9, \S 3.4]$ that $(X, T)$ is almost automorphic if $\phi: X \rightarrow X / S$ has some point $x$ with $\phi^{-1} \phi x=x$. It is easy to see that if $(X, T)$ is almost automorphic, then 
$\phi\left(x_{1}\right)=\phi\left(x_{2}\right)$ implies $\left(x_{1}, x_{2}\right) \in P$, whence $S \subset P$ and $S=P=Q$ (i.e., $(X, T)$ is proximally equicontinuous). We strengthen this by showing

(3.4) THEOREM. If $(X, T)$ is almost automorphic with $T$ abelian, then $(X, T)$ is locally almost periodic.

Proof. Choose $x \in X$ with $x S=\bigcap$ cls $x N(x, U) x N(x, U)^{-1}=\{x\}$. Let $U, V \in \mathscr{N}_{x}$ with $\bar{V} \subset U$. Using the filter base $\left\{x N(x, U) N(x, U)^{-1} \mid x \in X\right\}$, then

$$
x N(x, W) N(x, W)^{-1} \subset V
$$

for some open $W \in \mathscr{N}_{x}$. Thus, $W N(x, W)^{-1} \subset \operatorname{cls}(x N(x, W)) N(x, W)^{-1} \subset \bar{V} \subset U$, and $N(x, W)$ syndetic with $T$ abelian implies $N(x, W)^{-1}$ is syndetic. Hence $T$ is locally almost periodic at $x$.

Now choose $y \in X$ and $V \in \mathscr{N}_{y}$. Then $V t \in \mathscr{N}_{x}$ for some $t \in T$, and $W A \subset V t$ for some $W \in \mathscr{N}_{x}$ and $A$ syndetic by the above. Finally, $W s \in \mathscr{N}_{y}$ for some $s \in T$ and $(W s)\left(s^{-1} A t^{-1}\right) \subset V$, proving local almost periodicity at $y$.

The above result has been discovered independently by N. Markley (unpublished).

Note that since $\mathscr{A}$ almost automorphic implies $\mathscr{A} \subset \mathscr{K}$ the proof of (3.4) shows that $W A^{-1} \subset U$ with $A$ syndetic even when $T$ is nonabelian. Of course, it is not known whether $A^{-1}$ is syndetic. It would be interesting to know if (3.4) always holds. Also it is unknown whether proximal equicontinuity implies local almost periodicity if $T$ is abelian.

One can slightly generalize (3.4). Suppose $X$ is locally connected, $T$ is abelian, and $x S$ is finite for some $x \in X$. If $x S=\left\{x_{1}, \ldots, x_{n}\right\}$ and $V$ open in $\mathscr{N}_{x}$ with $x_{i} \in V t_{i}$, cls $\left(V t_{i}\right) \cap \operatorname{cls}\left(V t_{j}\right)=\varnothing$ if $i \neq j$, then $x N(x, U) N(x, U)^{-1} \subset \bigcup V t_{i}$ with $U$ connected in $\mathscr{N}_{x}$ implies that if $A=\{t \mid U t \subset \bar{V}\}, T=N(x, U)^{-1} K, C=\left\{t_{1}, \ldots, t_{n}\right\}$, then $N(x, U)^{-1} \subset A C$. Thus

(3.5) Corollary. Let $X$ be locally connected and T abelian. Suppose that $x S$ is finite for some $x \in X$. Then $(X, T)$ is locally almost periodic.

The following modifications enable us to remove the assumption of local connectivity. Suppose that $P$ is an equivalence relation and $x S=x P=\left\{x_{1}, \ldots, x_{n}\right\}$ for some $x \in X$. If $U \in \mathscr{N}_{x}$, then since the enveloping semigroup $E(X)$ has a unique minimal right ideal $I$, and $x_{1} p=x_{2} p=\cdots=x_{n} p$ for some $p \in I$, we have $x_{i} \in U t$ $(i=1, \ldots, n)$ for some $t \in T$, whence $x S \subset U t$. Proceeding as in (3.4), we have

(3.6) Corollary. Let $T$ be abelian. Suppose that $x S=x P$ is finite for some $x \in X$, and $P$ is an equivalence relation. Then $(X, T)$ is locally almost periodic.

For a related result in the nonabelian case, see [1a, Theorem 4].

4. The relativized case. In this situation, we are concerned with $\mathscr{F} \subset \mathscr{A}, \mathscr{F}$ a $T$-subalgebra. Instead of $\mathscr{E}$ and $\mathscr{D}$, we consider $\mathscr{F} \#$, the maximal almost periodic 
extension of $\mathscr{F}$ and $\mathscr{F} *$, the maximal distal extension of $\mathscr{F}$; moreover

$$
R(\mathscr{A}: \mathscr{A} \cap \mathscr{F} \#)
$$

plays the role of $S$. Under the assumption $g(\mathscr{F} \#)=F^{\#}=H(F, \mathscr{F} *) \subset H(F, \mathscr{A})$, the relativized version of (B) is $R(\mathscr{A}: \mathscr{A} \cap \mathscr{F} \#)=\{(p, q) \mid(p \omega, q \omega) \in Q(\mathscr{A}: \mathscr{F})$ $\left(\omega^{2}=\omega \in K, K\right.$ minimal ideal in $\left.\left.\beta T\right)\right\} \cap R(\mathscr{A}: \mathscr{F})$. For details, see [2, 14.23].

(4.1) THEOREM. With the above assumptions,

$$
R(\mathscr{A}: \mathscr{A} \cap \mathscr{F})=P(\mathscr{A}: \mathscr{F}) Q(\mathscr{A}: \mathscr{F}) .
$$

Proof. Since $R(\mathscr{A}: \mathscr{A} \cap \mathscr{F} \#)$ is a closed invariant equivalence relation containing $Q(\mathscr{A}: \mathscr{F})[2,14.22]$, it follows that $P(\mathscr{A}: \mathscr{F}) Q(\mathscr{A}: \mathscr{F}) \subset R(\mathscr{A}: \mathscr{A} \cap \mathscr{F})$. Now let $(p, q) \in R(\mathscr{A}: \mathscr{A} \cap \mathscr{F})$. Choose a minimal idempotent $\omega$ for which $q \omega=q$. Now $p|\mathscr{F}=q| \mathscr{F}=q \omega|\mathscr{F}=p \omega| \omega$. Since $(p, p \omega) \in P(\mathscr{A}),(p, p \omega) \in P(\mathscr{A}: \mathscr{F})$ and $(p, q)=(p, p \omega)(p \omega, q \omega) \in P(\mathscr{A}: \mathscr{F}) Q(\mathscr{A}: \mathscr{F})$. The result follows.

(4.2) Corollary. With the above assumptions, $R(\mathscr{A}: \mathscr{A} \cap \mathscr{F} \#)=Q(\mathscr{A}: \mathscr{F})^{2}$.

Proof. $Q(\mathscr{A}: \mathscr{F})^{2} \subset R(\mathscr{A}: \mathscr{A} \cap \mathscr{F} \#)=P(\mathscr{A}: \mathscr{F}) Q(\mathscr{A}: \mathscr{F}) \subset Q(\mathscr{A}: \mathscr{F})^{2}$.

The questions concerning general conditions when $F \# \subset H(F, \mathscr{A})$ and when $R(\mathscr{A}: \mathscr{A} \cap \mathscr{F} \#)=Q(\mathscr{A}: \mathscr{F})$ appear more difficult than in the absolute case. One case is clear: when $\mathscr{F} \leqq \mathscr{A}$, i.e., $\mathscr{A}$ is a distal extension of $\mathscr{F}$. For then $\mathscr{A} \subset \mathscr{F} *$ implies $F^{\#}=H(F, \mathscr{F} *) \subset H(F, \mathscr{A})$, and $P(\mathscr{A}: \mathscr{F})=\Delta$, the diagonal of $|\mathscr{A}| \times|\mathscr{A}|$, implies $R(\mathscr{A}: \mathscr{A} \cap \mathscr{F})=\Delta Q(\mathscr{A}: \mathscr{F})=Q(\mathscr{A}: \mathscr{F})$. It also follows by a similar proof to (2.18) that the relativized version of (B) holds iff

$$
R(\mathscr{A}: \mathscr{A} \cap \mathscr{F})=P(\mathscr{A}: \mathscr{F}) Q(\mathscr{A}: \mathscr{F}) .
$$

\section{REFERENCES}

1. J. P. Clay, Proximity relations in transformation groups, Trans. Amer. Math. Soc. 108 (1963), 88-96. MR 27 \#4218.

1a. J. Auslander and B. Horelick, Regular minimal sets. II, Compositio Math. 22 (1970), 203-214.

2. R. Ellis, Lectures on topological dynamics, Benjamin, New York, 1969.

3. R. Ellis and W. Gottschalk, Homomorphisms of transformation groups, Trans. Amer. Math. Soc. 94 (1960), 258-271. MR 23 \#A960.

4. H. Furstenberg, The structure of distal flows, Amer. J. Math. 85 (1963), 477-515. MR 28 \#602.

5. H. Keynes, The structure of weakly mixing minimal transformation groups, Illinois J. Math. (to appear).

6. H. Keynes and J. Robertson, Eigenvalue theorems in topological transformation groups, Trans. Amer. Math. Soc. 139 (1969), 359-369. MR 38 \#6029.

7. A. W. Knapp, Funetions behaving like almost automorphic functions, Proc. Sympos. Topological Dynamics (Colorado State Univ., Ft. Collins, Colo., 1967), Benjamin, New York, 1968, pp. 299-317. MR 38 \#6570. 
8. K. Peterson, Prime flows, disjointness, and mixing, Thesis, Yale University, New Haven, Conn., 1969.

9. W. A. Veech, Almost automorphic functions on groups, Amer. J. Math. 87 (1965), 719-751. MR 32 \#4469.

10. - The equicontinuous structure relation for minimal abelian transformation groups, Amer. J. Math. 90 (1968), 723-732. MR 38 \#702.

11. - Minimal transformation groups with distal points, Bull. Amer. Math. Soc. 75 (1969), 481-486.

UNIVERSTTY OF MINNESOTA, MINNEAPOLIS, MINNESOTA 55455 